\title{
Can higher order curvature theories explain rotation curves of galaxies?
}

\author{
S. Capozziello, V.F. Cardone ${ }^{\dagger}$ S. Carloni $;$ A. Troisi ${ }^{\S}$ \\ Dip. di Fisica "E.R. Caianiello", Universitá di Salerno \\ and Istituto Nazionale di Fisica Nucleare, Sez. di Napoli, Gruppo Coll. di Salerno \\ Via S. Allende, I-84081 Baronissi (Sa), Italy.
}

October 1, 2018

\begin{abstract}
Higher order curvature gravity has recently received a lot of attention due to the fact that it gives rise to cosmological models which seem capable of solving dark energy and quintessence issues without using "ad hoc" scalar fields. In this letter, a gravitational potential is obtained which differs from the Newtonian one because of a repulsive correction increasing with distance. We evaluate the rotation curve of our Galaxy and compare it with the observed data in order both to test the viability of these theories and to estimate the scalelength of the correction. It is remarkable that the Milky Way rotation curve is well fitted without the need of any dark matter halo and a similar result tentatively holds also for other galaxies.
\end{abstract}

PACS number(s): 04.50.+h, 98.80.-k, 98.35.df, 95.35.+d

*capozziello@sa.infn.it

†winny@na.infn.it

†carloni@sa.infn.it

§antro@sa.infn.it 


\section{Introduction}

In the last few years a very significant bulk of data have been accumulated from different observational campaigns leading to an unexpected picture of the universe. The Hubble diagram of type Ia Supernovae (SNeIa) [1] and the precise measurements of the anisotropy spectrum of the cosmic microwave background radiation (CMBR) by the BOOMERanG, MAXIMA and WMAP collaborations [2] strongly suggest that the universe is spatially flat and undergoing accelerating expansion driven by an unknown kind of dark energy. In fact, when combined with the data for the matter density parameter $\Omega_{M}$, these results lead to the conclusion that the contribution $\Omega_{X}$ of the dark energy is the dominant one, being $\Omega_{M} \simeq 0.3, \Omega_{X} \simeq 0.7$. On the other hand, astrophysical observations on galactic scales indicate that the luminous matter is unable to give account of the experimental rotation curves [3]. From a theoretical point of view this phenomenon is usually referred to the existence of the so called dark matter. Its nature is still matter of debate with different explanations ranging from baryonic MACHOs to cold dark matter particles [4].

Recently, in the cosmological framework, much attention has been devoted to higher order theories of gravity $[6,5,7,8,9,10,11]$. The reasons to take into account such teories come from fundamental physics where higher order curvature terms in the effective action of gravity are the results of interactions of scalar and graviton fields on curved spacetimes [12]. Besides, in this framework, several successful inflationary models have been formulated solving the shortcomings of cosmological standard model $[13,14]$.

In $[6,5,7]$ and then in $[8,9,10,11]$, it has been showed that it is possible to obtain the observed accelerating dynamics of universe expansion by taking into account higher order curvature terms into the gravitational Lagrangian. Furthermore, in [7], a successful test with SNeIa data has been performed. Having tested such a scheme on cosmological scales, it is straightforward to try to complement the approach by analyzing the low energy limit of these theories in order to see whether this approach is consistent with the local (i.e. on galactic scale) physics. This is the aim of this letter where we study the exterior space-time geometry generated by a spherically symmetric point-like source, in the framework of higher order theories assuming that the gravity Lagrangian contains a power law of Ricci scalar (i.e. $\mathcal{L} \propto f_{0} R^{n}$ ). We find that, in the weak field limit, the Newtonian potential is modified by an additive term which scales with the distance $r$ as a power law depending on $n$. Having obtained the corrected gravitational potential, we then theoretically evaluate the rotation curve of our Galaxy and compare it with the observed data. This test shows that the correction term allows to well fit the Milky Way

rotation curve without the need of dark matter. These results suggest that considering higher order gravitational theories can provide both an explanation to dark energy and dark matter. 


\section{Exact vacuum solution near a spherically symmet- ric source}

We study the low energy limit of higher order theories to verify if such a scheme is in agreement with astrophysical observations on galactic scales. We refer to the field equations obtained in $[6,5,7]$. They are:

$$
\begin{aligned}
G_{\alpha \beta}= & \frac{1}{f^{\prime}(R)}\left\{\frac{1}{2} g_{\alpha \beta}\left[f(R)-R f^{\prime}(R)\right]+\right. \\
& \left.+f^{\prime}(R)^{; \mu \nu}\left(g_{\alpha \mu} g_{\beta \nu}-g_{\alpha \beta} g_{\mu \nu}\right)\right\},
\end{aligned}
$$

the prime denoting derivatives with respect to $R$. These field equations can be decomposed into the time-time component and the trace which can be combined in a simpler relation. Assuming $f(R)=f_{0} R^{n}$, as in $[5,6,7]$, we obtain:

$$
R_{t t}=\left(\frac{2 n-1}{6 n}\right) g_{t t} R
$$

depending explicitly on the exponent $n$ of the theory.

Note that the off-diagonal Einstein equations reduces to identities for the metric that we are going to use here, which is

$$
d s^{2}=-\frac{1}{K(r)} d t^{2}+K(r) d r^{2}+r^{2} d \theta^{2}+r^{2} \sin \theta d \phi^{2},
$$

where the spherical symmetry is assumed. Inserting it into Eq.(2), we get an equation for $K(r)$ that can be analytically solved. We find:

$$
\begin{gathered}
K(r)=C_{1} r^{\alpha(n)}+C_{2} r^{\beta(n)}, \\
\alpha(n)=\sqrt{\frac{4 n-1}{2(n-1)}} \times[\mathcal{P}(n)-\mathcal{Q}(n)], \\
\beta(n)=\sqrt{\frac{4 n-1}{2(n-1)}} \times[\mathcal{P}(n)+\mathcal{Q}(n)], \\
\mathcal{P}(n)=-\frac{3}{2}\left(\frac{3 n-1}{4 n-1}\right) \sqrt{\frac{2(4 n-1)}{n-1}} \\
\mathcal{Q}(n)=\sqrt{\mathcal{P}^{2}(n)-4}
\end{gathered}
$$

We have now all what we need to obtain the gravitational potential $\Psi(r)$ generated by a point-like source. To this aim, one only needs to remember that it is $g_{r r}=1+2 \Psi / c^{2}$, so that we immediately get: 


$$
\Psi(r)=\frac{c^{2}}{2}\left[C_{1} r^{\alpha(n)}+C_{2} r^{\beta(n)}\right] .
$$

where we have opportunely chosen the additive arbitrary constant. Up to now, the two integration constants $C_{1}$ and $C_{2}$ are completely arbitrary, but some general considerations help in choosing their values. First, we note that, in order to get the correct physical dimensions for $\Psi, C_{1}$ and $C_{2}$ must have the dimensions of $l^{-\alpha(n)}$ and $l^{-\beta(n)}$, being $l$ a length. It is, thus, convenient to redefine them as:

$$
C_{1}=\frac{s_{1}}{\xi_{1}^{\alpha(n)}} \quad, \quad C_{2}=\frac{s_{2}}{\xi_{2}^{\beta(n)}}
$$

being $s_{i}$ the sign of $C_{i}$ and $\left(\xi_{1}, \xi_{2}\right)$ two undetermined scalelengths. Let us now consider the behaviour of the two powers $(\alpha, \beta)$ in Eq.(9) with respect to the exponent $n$. A careful analysis shows that:

$$
\begin{aligned}
& \text { for } n \in(0.25,1) \quad \alpha(n) \simeq-1, \quad \beta(n)>0 ; \\
& \text { for } n \notin(0.25,1) \quad \alpha(n)<0, \quad \beta(n) \simeq-1 .
\end{aligned}
$$

Let us take into account the case $n \in(0.25,1)$. The first term in Eq.(9) is the leading one for small $r$, i.e. for $r<<\xi_{2}$. Actually, we know that on small scale (at least for distances within the Solar System) the gravitational potential is Newtonian, i.e. it is attractive and scales as $1 / r$. In order to recover this behaviour, we thus need that $s_{1}=-1$, while we have no constraints on $s_{2}$. The case $s_{2}<0$ may be excluded, since the corresponding gravitational force between two point masses should be more and more attractive as the bodies are far away and this is not a physical case. We may thus conclude that, for $n \in(0.25,1)$, it is $s_{1}=-1$ and $s_{2}=+1$.

Similar considerations show that the case $n \notin(0.25,1)$ is unphysical. For these values of $n$, both terms in Eq.(9) are present on small scales. In order to recover the Newtonian potential on such scales, we have to impose $s_{2}=-1$. On the other side, if $s_{1}$ were equal to 1 , then we will obtain a repulsive correction to the gravitational force on small scales leading to a result contrasting with observations. The same contradiction will be present if $s_{1}=-1$ since, in this case, the gravitational force should be more attractive than as probed by many experiments. We have thus to conclude that models with $n \notin(0.25,1)$ are not physically acceptable since their low energy limit does not reproduce the well known Newtonian potential on Solar System scales.

From now on, we will only consider models with $n$ in the range $(0.25,1)$ and rewrite the gravitational potential of a pointlike source with mass $M$ as:

$$
\Psi(r)=-\frac{c^{2}}{2}\left[\left(\frac{r}{\xi_{1}}\right)^{-1}-\left(\frac{r}{\xi_{2}}\right)^{\beta(n)}\right] .
$$


A first estimate of $\xi_{1}$ may be obtained observing that, for $r<<\xi_{2}$, Eq.(11) reduces to

$$
\Psi(r) \sim-\frac{c^{2}}{2}\left(\frac{r}{\xi_{1}}\right)^{-1} .
$$

Since we have to recover the Newtonian potential at these scales, we have to fix:

$$
\xi_{1}=\frac{2 G M}{c^{2}} \simeq 9.6 \times \frac{M}{M_{\odot}} \times 10^{-17} \mathrm{kpc},
$$

with $M_{\odot}$ the mass of the Sun. The value of $\xi_{2}$ is a free parameter of the theory. Up to now, we can only say that $\xi_{2}$ should be much larger than the Solar System scale in order not to violate the constraints coming from local gravity experiments.

\section{The Milky Way rotation curve}

Eq.(11) gives the gravitational potential of a pointlike source. Since real galaxies are not pointlike, we have to generalize Eq.(11) to an extended source. To this aim, we may suppose to divide the Milky Way in infinitesimal mass elements, to evaluate the contribution to the potential of each mass element and then to sum up these terms to get the final potential. Assuming spheroidal symmetry and using Eq.(9), the gravitational potential generated by a galaxy is ${ }^{1}$ :

$$
\Psi(R, z)=\frac{c^{2}}{2}\left[\Psi_{1}(R, z)+\Psi_{2}(R, z)\right],
$$

with

$$
\Psi_{i}=C_{i} \int_{0}^{\infty} R^{\prime} d R^{\prime} \int_{0}^{2 \pi} d \phi^{\prime} \int_{-\infty}^{+\infty} d z^{\prime} \rho\left(R^{\prime}, z^{\prime}\right) r^{\eta_{i}},
$$

being $\eta_{i}=-1(\beta)$, for $i=1(2)$, while $\rho(R, z)$ is the mass density of the galaxy (having assumed spheroidal symmetry). We remark that we have adopted cylindrical coordinates $(R, \phi, z)$ so that it is:

$$
r^{2}=\left|\mathbf{r}-\mathbf{r}^{\prime}\right|^{2}=R^{2}+R^{\prime 2}-2 R R^{\prime} \cos \phi^{\prime}+\left(z-z^{\prime}\right)^{2} .
$$

In order to restore the correct dimensions of the potential, we have to recast the two constants $C_{1}$ and $C_{2}$ in a way similar to Eq.(10). It is :

$$
C_{1}=-\frac{2 G}{c^{2} \xi_{1}^{\alpha(n)+1}} \quad, \quad C_{2}=\frac{2 G}{c^{2} \xi_{2}^{\beta(n)+1}} .
$$

A simple check shows that, with this assumption, the potential $\Psi_{i}$ in Eq.(13) has the correct physical dimensions. Note also that, since $\alpha=-1$, the exact value of $\xi_{1}$ is

\footnotetext{
${ }^{1}$ In this section, $R$ is the radial coordinate, not the Ricci scalar.
} 
meaningless and hence $\left(n, \xi_{2}\right)$ are the only parameters to determine the shape of the gravitational potential for an extended source. The rotation curve may then be obtained by the standard rule:

$$
v_{c}^{2}(R)=\left.R \frac{\partial \Psi}{\partial R}\right|_{z=0} .
$$

To test whether the theory is in agreement with observations and to determine the parameter $\xi_{2}$, we have computed the Milky Way rotation curve modeling our Galaxy as a two components system, a spheroidal bulge and a thin disk. In particular, following [15], we assume:

$$
\begin{gathered}
\rho_{\text {bulge }}=\rho_{0}\left(\frac{m}{r_{0}}\right)^{-1.8} \exp \left(-\frac{m^{2}}{r_{t}^{2}}\right), \\
\rho_{\text {disk }}=\frac{\Sigma_{0}}{2 z_{d}} \exp \left(-\frac{R}{R_{d}}-\left|\frac{z}{z_{d}}\right|\right)
\end{gathered}
$$

with $m^{2}=R^{2}+z^{2} / q^{2}$. The central densities $\rho_{0}$ and $\Sigma_{0}$ are conveniently related to the bulge total mass $M_{\text {bulge }}$ and the local surface density $\Sigma_{\odot}$ by the following two relations:

$$
\rho_{0}=\frac{M_{\text {bulge }}}{4 \pi q \times 1.60851}, \Sigma_{0}=\Sigma_{\odot} \exp \left(\frac{R_{0}}{R_{d}}\right),
$$

being $R_{0}=8.5 \mathrm{kpc}$ the distance of the Sun to the Galactic Centre. Following [15], we fix the Galactic parameters as follows:

$$
\begin{gathered}
M_{\text {bulge }}=1.3 \times 10^{10} \mathrm{M}_{\odot}, r_{0}=1.0 \mathrm{kpc}, r_{t}=1.9 \mathrm{kpc}, \\
\Sigma_{\odot}=48 \mathrm{M}_{\odot} \mathrm{pc}^{-2}, R_{d}=0.3 R_{0}, z_{d}=0.18 \mathrm{kpc} .
\end{gathered}
$$

The Milky Way rotation curve $v_{c}(R)$ can be reconstructed starting from the data on the observed radial velocities $v_{r}$ of test particles. We follow [15] reconstructing the rotation curve from the data on H II regions and molecular clouds in [16] and those on a sample of classical Cepheids in the outer disc obtained by Pont et al. [17].

For a given $n$, we perform a $\chi^{2}$ test to see whether the modified gravitational potential is able to fit the observed rotation curve and to constrain the value of $\xi_{2}$. Since a priori we do not know what is the range for $\xi_{2}$, we get a first estimate of $\xi_{2}$ by a simple approach. For a given $R$, we compute $\xi_{2}$ imposing that the theoretical rotation curve is equal to the observed one. Then, we study the distribution of the $\xi_{2}$ values thus obtained and evaluate both the median $\xi_{2}^{\text {med }}$ and the median deviation $\delta \xi_{2}$. The usual $\chi^{2}$ test is then performed with the prior that $\xi_{2}$ lies in the range $\left(\xi_{2}^{m e d}-5 \delta \xi_{2}, \xi_{2}^{\text {med }}+5 \delta \xi_{2}\right)$. As a first test, we arbitrarily fix $n=0.35$. We get:

$$
\xi_{2}=14.88 \mathrm{kpc}, \chi^{2}=0.96
$$




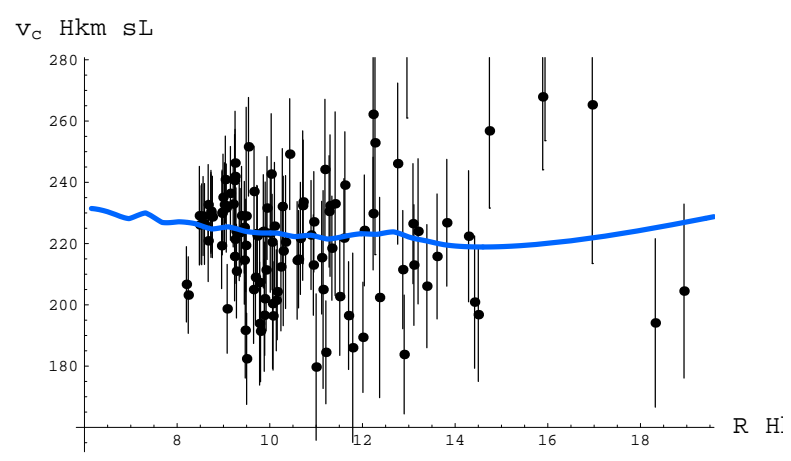

Figure 1: Observed data and theoretical Milky Way rotation curve computed using the modified gravitational potential with $n=0.35$ and $\xi_{2}=14.88 \mathrm{kpc}$. Note that the points with $R$ between 15.5 and $17.5 \mathrm{kpc}$ are likely affected by systematic errors as discussed in [17].

In Fig. 1, we show both the theoretical rotation curve for $\left(n, \xi_{2}\right)=(0.35,14.88)$ and the observed data. The agreement is quite good even if we have not added any dark matter component to the Milky Way model. This result seems to suggest that our modified theory of gravitation is able to fit galaxy rotation curves without the need of dark matter. As a final remark, we note that $\xi_{2}^{m e d}=14.37 \mathrm{kpc}$ that is quite similar to the best fit value. Actually, a quite good estimate is also obtained considering the value of $\xi_{2}$ evaluated using the observed rotational velocity at $R_{0}$. This suggest that a quick estimate of $\xi_{2}$ for other values of $n$ may be directly obtained imposing $v_{c, \text { theor }}\left(R_{0} ; n, \xi_{2}\right)=v_{c, \text { obs }}\left(R_{0}\right)$.

\section{Conclusions}

In this letter, it has been analyzed the low energy limit of higher order theories of gravity considering a power law function of Ricci scalar for the gravitational Lagrangian. An exact solution of the field equation has been obtained. The resulting gravitational potential for a point-like source is the sum of a Newtonian term and a contribute whose rate depends on a function of the exponent $n$ of Ricci scalar. The potential agrees with experimental data if $n$ ranges into the interval $(0.25,1)$, so that the correction term scales as $r^{\beta}$ with $\beta>0$.

The following step is the generalization of this result to an extended source as a galaxy. To this aim the experimental data and the theoretical prediction for the rotation curve of Milky Way have been compared. The final result has been that the modified potential is able to provide a rotation curve which fits data without adding any dark matter component. This result has to be tested further before drawing a definitive conclusion against the need for galactic dark matter. To this aim, one must show that a potential like that predicted by our model is able to fit rotation curves of a homogeneous 
sample of external galaxies with both well measured rotation curves and detailed surface photometry. In particular, the exponent $n$ coming out from the fit must be the same for all the galaxies, while $\xi_{2}$ could be different being related to the scale where deviations from the Newtonian potential sets in. On the other hand, however, it is worth noting that for $n=1 / 3$, it is $\beta=1$. Such kind of modified potential has been taken into account (on the basis of a different theory) by Mannheim [18] who has successfully fitted the rotation curves of external galaxies. He has also found that the normalization parameter (which plays in his theory the same role as $\xi_{2}$ ) changes from one galaxy to another in agreement with our expectations. This is an encouraging result since it states that our model could be able to work correctly at least for one value of the exponent $n$.

It is worth noting that modifications of the gravitational potential are not the only way to fit galactic rotation curves without adding any dark matter components. A quite successful scheme is that adopted in the MOND theory [19] where it is the acceleration law to be changed. However, it is worth to stress that our model is completely different from the MOND approach. MOND has been originally proposed as a phenomelogical scheme to solve an astrophysical problem and it cannot be derived from a Lagrangian field theory (even if some tentatives have been done). On the contrary, our modified gravitational potential is the natural outcome of a Lagrangian theory and it is thus physically well founded. Besides, contrary to MOND, our model has also a cosmological counterpart $[5,6,7]$.

In this paper, we have presented an indication that it is possible to reduce the dark energy and dark matter issues under the same higher order theories of gravity. In fact, this can give rise to realistic models working at very large scales (cosmology) and local scales (galaxies).

\section{References}

[1] Riess A.G. et al., AJ, 116, 1009 ,1998; Perlmutter S. et al., ApJ, 517, 565, 1999; Tonry J.L. et al., astro - ph/0305008, 2003; Knop R.A. et al., astro-ph/0309368.

[2] de Bernardis P. et al., Nature, 404, 955, 2000; Stompor R. et al. ApJ, 561, L7, 2001; Spergel D.N. et al., astro - ph/0302209, 2003.

[3] Sofue Y., Rubin V., ARA\&A, 39, 137, 2001.

[4] Olive K.A., astro-ph/0301505, 2003.

[5] Capozziello S., Carloni S., Troisi A., "Recent Research Developments in Astronomy and Astrophysics"-RSP/AA/21-2003 (astro-ph/0303041).

[6] Capozziello S., Int. J. Mod. Phys. D 11, 483, 2002.

[7] Capozziello S., Cardone V.F., Carloni S., Troisi A., Int. J. Mod. Phys. D 12, 1969, 2003. 
[8] Carroll S., Duvvuri V., Trodden M., Turner M.S., astro - ph/0306438, 2003.

[9] Vollick D., Phys. Rev. D 68, 063510, 2003.

[10] Nojiri S., Odintsov S.D., Phys. Rev. D 68, 123512, 2003.

[11] Allemandi G., Borowiec A., Francaviglia M., hep - th/0403264, 2004.

[12] Birrell N. and Davies P., Quantum Fields in Curved Space, Cambridge Univ. Press, Cambridge 1982.

[13] Starobinsky A.A., Phys. Lett. B 91, 99, 1980.

[14] Mijic M.B., Morris M.S. and Suen W., Phys. Rev. D 34, 2934, 1986.

[15] Dehnen W., Binney J., MNRAS, 294, 429, 1998.

[16] Brand J., Blitz L., A\&A, 275, 67, 1993.

[17] Pont F. et al., A\&A, 318, 416, 1997.

[18] Mannheim P.D., ApJ, 419, 150, 1993.

[19] Milgrom M., ApJ, 270, 365, 1983; Sanders R.H., McGaugh S., ARA\&A, 40, 263, 2002. 